\title{
Pembrolizumab plus platinum-based chemotherapy for squamous non-small cell lung cancer: the new kid on the block
}

\author{
Kimberley Hockenhull $^{1} \wedge$, Ana Ortega-Franco ${ }^{1}$, Raffaele Califano ${ }^{1,2,3}$ \\ ${ }^{1}$ Department of Medical Oncology, The Christie NHS Foundation Trust, Manchester, UK; ${ }^{2}$ Department of Medical Oncology, Manchester \\ University NHS Foundation Trust, Manchester, UK; ${ }^{3}$ Division of Cancer Sciences, University of Manchester, Manchester, UK \\ Correspondence to: Dr. Raffaele Califano. Department of Medical Oncology, The Christie NHS Foundation Trust, 550 Wilmslow Road, Manchester, \\ M20 4BX, UK. Email: raffaele.califano@christie.nhs.uk. \\ Comment on: Paz-Ares L, Luft A, Vicente D, et al. Pembrolizumab plus Chemotherapy for Squamous Non-Small-Cell Lung Cancer. N Engl J Med \\ 2018;379:2040-51.
}

Submitted Jun 03, 2021. Accepted for publication Sep 02, 2021.

doi: $10.21037 /$ tlcr-20-715

View this article at: https://dx.doi.org/10.21037/tlcr-20-715

\section{Introduction}

Immune checkpoint inhibitors (ICIs) and targeted therapies have revolutionised the diagnostic and treatment paradigm of advanced non-small cell lung cancer (NSCLC). However, treatment advances in squamous cell subtype have been much slower than those occurring in adenocarcinoma, mainly due to the lack of targetable oncogenic aberrations in squamous tumours. The unprecedented durable response and favourable toxicity profile observed with ICIs in advanced squamous NSCLC, represents a true therapeutic milestone in a population previously limited to cytotoxic chemotherapy (Cht).

The inhibition of programmed cell death ligand 1 (PD1/PD-L1) pathway has been extensively investigated in NSCLC and currently dominates the treatment landscape of advanced NSCLC. The first approval of ICIs came from several randomized trials conducted in platinumtreated advanced NSCLC patients (any histology) (1-4) where overall survival (OS) and toxicity profile favoured PD-1/PD-L1 inhibitors over docetaxel. Subsequently, KEYNOTE-024 (5) showed that, in advanced NSCLC patients (all histologies) with a PD-L1 tumour proportion score (TPS) $\geq 50 \%$, first-line pembrolizumab achieved higher objective response rates (ORR) and longer progression-free survival (PFS) and OS when compared to standard platinum-based Cht. However, high
PD-L1 expression only occurs in a third of NSCLC patients and, until recently, platinum-based Cht remained the only approved option for fit patients with a PD-L1 TPS $<50 \%$.

In the phase III trial KEYNOTE-407 (6), 559 patients with treatment-naïve advanced squamous NSCLC and performance status $0-1$ were randomised to receive 4 cycles of carboplatin (AUC 6, 3 weekly) with either Paclitaxel $\left(200 \mathrm{mg} / \mathrm{m}^{2}, 3\right.$ weekly) or nab-paclitaxel $\left(100 \mathrm{mg} / \mathrm{m}^{2}\right.$ on day 1, 8 and 15) plus Pembrolizumab (200 mg, 3 weekly) or placebo for 35 cycles. Stratification factors were PD-L1 TPS $\geq 1 \%$ versus $<1 \%$ assessed by $222 \mathrm{C} 3$ assay, type of taxane (paclitaxel versus nab-paclitaxel) and geographic region (East Asia versus rest of the world). The co-primary endpoints were PFS and OS.

After a median follow-up of 7.8 months, the chemoimmunotherapy arm achieved longer PFS (4.8 vs. 6.4 months, HR 0.56; 95\% CI, 0.45-0.70; $\mathrm{P}<0.0001)$ and OS (15.9 vs. 11.3 months, HR 0.64; 95\% CI, 0.49-0.85; $\mathrm{P}<0.001)$. At progression, $42.8 \%$ of the patients randomized to the control arm received ICI (75 patients crossed over to Pembrolizumab and 12 more received ICI as subsequent line). The benefit in OS and PFS was consistent across all PD-L1 subgroups (TPS $<1 \%, 1-49 \%$ and $>50 \%$ ). Incidence of grade $\geq 3$ adverse events (AEs) (69.8\% vs. 68.2\%) and AEs leading to death $(8.3 \%$ vs. $6.4 \%)$, was similar between the two arms. According to an exploratory analysis (7),

^ ORCID: 0000-0003-0482-785X. 
the type of taxane (paclitaxel versus nab-paclitaxel) did not impact in treatment tolerance or treatment efficacy in terms of OS, PFS and ORR. Furthermore, the addition of Pembrolizumab to chemotherapy maintained or improved Health-related quality of life (HRQoL) measurements relative to baseline. There was also improved HRQoL at weeks 9 and 18 versus chemotherapy alone (8). On the basis of KEYNOTE-407, the combination of carboplatin and (nab)paclitaxel plus pembrolizumab is now approved by FDA and EMA as a first line option for advanced squamous NSCLC patients, independently on PD-L1 expression.

\section{Putting KEYNOTE-407 into context}

KEYNOTE-024 (5) compared pembrolizumab (200 mg 3 weekly for up to 35 cycles) to standard platinum-based chemotherapy in untreated NSCLC patients with PD-L1 $\geq 50 \%$. Patients were ALK and EGFR negative and included squamous $(\mathrm{n}=56)$ and non-squamous $(\mathrm{n}=249)$ subtypes. The choice of platinum-based chemotherapy (4-6 cycles) was at investigators' discretion. The most common chemotherapy combination was carboplatin and pemetrexed $(n=67)$, however other regimes included cisplatin/pemtrexed $(n=36)$, carboplatin/gemcitabine $(\mathrm{n}=20)$, cisplatin/gemcitabine $(n=11)$ and carboplatin/paclitaxel $(n=17)$. Crossover to pembrolizumab was permitted at progression for patients treated with standard chemotherapy upon meeting eligibility criteria. In addition, maintenance therapy with pemtrexed following platinum-pemtrexed chemotherapy was permitted in non-squamous patients. Primary end point was PFS and secondary end point was OS. At the second pre-planned interim analysis (median follow-up: 11.2 months), pembrolizumab was associated with longer PFS (HR 0.50; 95\% CI, 0.37-0.68; $\mathrm{P}=0.001$ ) and OS (HR $0.60 ; 95 \%$ CI, 0.41-0.89; $\mathrm{P}=0.005)$. Median OS was not reached (NR) in either arm. An updated analysis, with a median follow-up of 25.2 months, confirmed a longer OS (30 vs. 14.2 months, HR 0.63; 95\% CI, 0.47-0.86) in the pembrolizumab arm. Hazard ratio for disease progression or death in squamous cell carcinoma was 0.35 (95\% CI, 0.17-0.71) vs. 0.55 (95\% CI, 0.39-0.76) compared to 0.55 (95\% CI, 0.39-0.76) in non-squamous histology, indicating efficacy of pembrolizumab in both groups. Given the OS and PFS benefits observed in KEYNOTE-024, pembrolizumab remains the only checkpoint inhibitor approved in the first-line setting as monotherapy for patients with $\mathrm{PD}-\mathrm{L} 1 \geq 50 \%$.

KEYNOTE-042 (9) was conducted to evaluate the efficacy of pembrolizumab in 3 different PD-L1 expression brackets $(\geq 50 \%, \geq 20 \%$ and $\geq 1 \%)$. Treatment naïve advanced NSCLC patients (ALK/EGFR negative) with a PD-L1 expression of $\geq 1 \%$ were randomized to receive pembrolizumab and carboplatin plus paclitaxel (squamous cell histology) or carboplatin plus pemetrexed (non-squamous histology). Pembrolizumab demonstrated a significant improvement in OS across all three PD-L1 thresholds: TPS $\geq 50 \%$ : 20 vs. 12.2 months (HR 0.69; $95 \%$ CI, 0.56-0.85, $\mathrm{P}=0.0003)$, TPS $\geq 20 \%: 17.7$ vs. 13 months (HR 0.77; 95\% CI, 0.64-0.92, $\mathrm{P}=0.0020$ ), TPS $\geq 1 \%$ : 16.7 vs. 12.1 months (HR 0.81; 95\% CI, 0.71-0.93, $\mathrm{P}=0.0018)$. An exploratory analysis for patients with a PD-L1 expression of $1-49 \%$ did not show a difference in OS between the two arms (13.4 vs. 12.1 months, HR 0.92; $95 \%$ CI, 0.77-1.11). Crossover from pembrolizumab to ChT was not allowed in the protocol and therefore the OS benefit is likely driven by patients with a PD-L1 expression $\geq 50 \%$.

Notably, PFS was not significantly improved in the PD$\mathrm{L} 1 \geq 50 \%$ group and was therefore not tested in the other lower PDL1 expression subgroups. This was different from KEYNOTE-024 (5) which showed significant improvement in PFS in the pembrolizumab group, investigators concluded this may be due to lack of cross over to immunotherapy following progressive disease in this study and the geographical demographics of the patients [KEYNOTE-042 (9) was mainly Asia-Pacific, eastern European and South America whereas KEYNOTE-024 (5) was North American and Western Europe] which may have limited to availability of immunotherapy in the second line settings in the former groups.

IMpower-131 (10) evaluated unselected treatment naïve squamous advanced NSCLC patients who were randomised to receive either atezolizumab $(1,200 \mathrm{mg}$ three weekly) plus carboplatin (AUC 6)/paclitaxel $\left(200 \mathrm{mg} / \mathrm{m}^{2}\right)$ (Arm A - ACP) or atezolizumab plus carboplatin/nab-paclitaxel $\left(100 \mathrm{mg} / \mathrm{m}^{2}\right)(\mathrm{Arm} \mathrm{B}-\mathrm{ACnP})$ or carboplatin/nab-paclitaxel alone (Arm $\mathrm{C}-\mathrm{CnP}$ ). $\mathrm{CnP}+/$ - atezolizumab was the initial test setting. Pts received Cht \pm atezolizumab for 4 or 6 cycles as per investigator choice, followed by atezolizumab maintenance (Arms A and B). Co-primary endpoints were investigator-assessed PFS and OS in the intention to treat (ITT) population. PFS was longer in arm $\mathrm{B}$ with the addition of nab-paclitaxel (6.3 vs. 5.6 months, HR 0.71; CI, 0.60-0.85) but Interim analysis indicated no significant difference in OS with the addition of atezolizumab to CnP. However, in patients with high PDL-1 expression there was a longer OS 
in the chemo-immunotherapy arm (23.6 vs. 14.1 months, HR 0.56; CI, 0.32-0.99). More than $40 \%$ of patients in the chemotherapy arm, received immunotherapy as later line. This was not dissimilar to what reported for Keynote 407 (6) and therefore unlikely to be the cause of lack of OS benefit seen in IMpower 131 (10).

CHECKMATE $227(11,12)$ investigated the role of first-line nivolumab and nivolumab-based regimens in advanced NSCLC without sensitizing alterations, irrespective of tumour histology. Patients with a PD-L1 expression of $\geq 1 \% \quad(n=1,189)$ were enrolled in Part 1a of the study and randomized $(1: 1: 1)$ to receive nivolumab plus low dose ipilimumab ( $\mathrm{n}=396)$, histology based ChT $(n=397)$ or nivolumab monotherapy $(n=396)$. Part $1 b$ of the study recruited patients with $<1 \%$ PD-L1 expression $(\mathrm{n}=550)$ who were then randomized $(1: 1: 1)$ to either receive nivolumab plus ipilimumab $(\mathrm{n}=187)$, ChT $(\mathrm{n}=186)$ or ChT plus nivolumab ( $\mathrm{n}=177)$ (12). The independent coprimary endpoints were PFS in patients harboring tumors with a high tumour mutational burden (TMB) defined as $\geq 10$ mutations per megabase, irrespective of PDL1 expression level, and OS in patients with tumours expressing PD-L1 $\geq 1 \%$. $94.8 \%$ of the 1,739 randomized patients' tumour samples available for TMB assessment with $44.2 \%$ having at least $10 \mathrm{mut} / \mathrm{Mb}$. PFS was longer (7.2 vs. 5.4 months, HR 0.58 ; 95\% CI, 0.41-0.81; $\mathrm{P}=0.0002$ ) in the ICI-doublet compared to the ChT arm. The PFS benefit was consistent irrespective of PD-L1 expression and tumour histology. Updated results did not show a difference in OS when patients were stratified by TMB (13).

In patients with $\mathrm{PD}-\mathrm{L} 1 \geq 1 \%$, nivolumab plus ipilimumab demonstrated longer OS (17.1 vs. 14.9 months, HR 0.79; 97.72\% CI, 0.65-0.96, P=0.007) over ChT (14). Subgroup analysis based on \%PD-L1 expression showed OS benefit was only seen in those patients with $\geq 50 \%$ PD-L1 expression. Of note, the median duration of response was 23.2 months with nivolumab plus ipilimumab vs. 6.2 months with ChT. A remarkable proportion $(49.9 \%)$ of patients in the nivolumab-ipilimumab arm showed continued response at 2 years.

In part 2 of Checkmate 227 trial, patients with advanced NSCLC (any histology and unselected for PD-L1 expression), were randomised to receive histology-based chemotherapy with or without nivolumab (15). OS in the non-squamous population was the primary endpoint and was not met (18.8 vs. 15.6 months, HR 0.86; CI, 0.69-1.08, $\mathrm{P}=0.1859)$. A descriptive analysis for patients with squamous histology $(n=212)$, showed a longer OS in the chemo-IO arm (18.3 vs. 12 months, HR 0.69; CI, 0.50-0.97). There was no data from any biomarker analysis to suggest any potential reason for the differential effect seen in squamous patients. Notably, this analysis was underpowered and remains hypothesis generating.

\section{Conclusions}

The lack of head-to-head comparison makes it very difficult to determine which is the best immune checkpoint blockade strategy as first-line treatment of squamous NSCLC. Evidence from KEYNOTE-407 (6) suggests PFS and OS benefit with the addition of pembrolizumab to platinumtaxane chemotherapy and this was consistent across all PD-L1 subgroups (TPS $<1 \%, 1-49 \%$ and $>50 \%$ ). In IMpower131 (10), there was no OS benefit in the ITT population but patients with high PD-L1 expression (TC3 or IC3) appeared to derive an OS benefit with the addition of atezolizumab. Notably, the study was not powered for this subgroup analysis.

Trial interpretation is also made difficult due to the maintenance phase of $\mathrm{PD}(\mathrm{L})-1$ inhibition following initial platinum doublet. This is also confounded by switches in the control arms on progression to allow crossover to receive ICI in some of the above trials $(5,6,10,11)$. High levels of crossover make OS data potentially prone to error and this is not standardised across trials. Geographical location of the studies may indirectly limit access to second line immunotherapy which again may skew OS data, depending on the sites of recruitment, socioeconomic backgrounds of patients and type of healthcare system.

At present, the KEYNOTE407 (6) regimen represents the only first-line chemo-immunotherapy achieving OS benefit over standard chemotherapy for squamous NSCLC patients. This combination is certainly an option for fit patients with PD-L1 expression of $1-49 \%$ but further research is needed to identify which patients with PD-L1 $\geq 50 \%$ should receive chemo-immunotherapy instead of single agent pembrolizumab.

\section{Acknowledgments}

Funding: None.

\section{Footnote}

Provenance and Peer review: This article was commissioned by the editorial office, Translational Lung Cancer Research. 
The article did not undergo external peer review.

Conflicts of Interest: All authors have completed the ICMJE uniform disclosure form (available at https://dx.doi. org/10.21037/tlcr-20-715). RC reports - Honoraria and consultancy fees: AstraZeneca, Boeringher Ingelheim, Lilly Oncology, Roche, Pfizer, MSD, Bristol Myers Squibb, Takeda, Bayer, Ipsen and Novartis. Grants paid to Institution for conduct of clinical trials or contracted research: Roche, AstraZeneca, Pfizer, Clovis, Lilly Oncology, MSD, BMS, Abbvie, Takeda and Novartis. Stock Ownership: The Christie Private Care. Non-remunerated activities: Principal investigator for trials with Roche, AstraZeneca, Pfizer, Clovis, Lilly Oncology, MSD, BMS, Abbvie, Takeda and Novartis. Other non-remunerated membership: ESMO, EORTC. The other authors have no conflicts of interest to declare.

Ethical Statement: The authors are accountable for all aspects of the work in ensuring that questions related to the accuracy or integrity of any part of the work are appropriately investigated and resolved.

Open Access Statement: This is an Open Access article distributed in accordance with the Creative Commons Attribution-NonCommercial-NoDerivs 4.0 International License (CC BY-NC-ND 4.0), which permits the noncommercial replication and distribution of the article with the strict proviso that no changes or edits are made and the original work is properly cited (including links to both the formal publication through the relevant DOI and the license). See: https://creativecommons.org/licenses/by-nc-nd/4.0/.

\section{References}

1. Rittmeyer A, Barlesi F, Waterkamp D, et al. Atezolizumab versus docetaxel in patients with previously treated non-small-cell lung cancer (OAK): a phase 3, openlabel, multicentre randomised controlled trial. Lancet 2017;389:255-65.

2. Borghaei H, Paz-Ares L, Horn L, et al. Nivolumab versus Docetaxel in Advanced Nonsquamous Non-Small-Cell Lung Cancer. N Engl J Med 2015;373:1627-39.

3. Brahmer J, Reckamp KL, Baas P, et al. Nivolumab versus Docetaxel in Advanced Squamous-Cell Non-Small-Cell Lung Cancer. N Engl J Med 2015;373:123-35.

4. Herbst RS, Baas P, Kim DW, et al. Pembrolizumab versus docetaxel for previously treated, PD-L1-positive, advanced non-small-cell lung cancer (KEYNOTE-010): a randomised controlled trial. Lancet 2016;387:1540-50.

5. Reck M, Rodríguez-Abreu D, Robinson AG, et al. Pembrolizumab versus Chemotherapy for PD-L1Positive Non-Small-Cell Lung Cancer. N Engl J Med 2016;375:1823-33.

6. Paz-Ares L, Luft A, Vicente D, et al. Pembrolizumab plus Chemotherapy for Squamous Non-Small-Cell Lung Cancer. N Engl J Med 2018;379:2040-51.

7. Halmos B, Luft A, Majem M, et al. Choice of taxane and outcomes in the Keynote-407 study of Pembrolizumab plus Chemotherapy for metastatic squamous NSCLC. J Thorac Oncol 2018;13:S391.

8. Mazieres J, Kowalski D, Luft A, et al. Health-Related Quality of Life With Carboplatin-Paclitaxel or nabPaclitaxel With or Without Pembrolizumab in Patients With Metastatic Squamous Non-Small-Cell Lung Cancer. J Clin Oncol 2020;38:271-80.

9. Lopes G, Wu YL, Kudaba I, et al. Pembrolizumab (pembro) versus platinum-based chemotherapy (chemo) as first-line therapy for advanced/metastatic NSCLC with a PD-L1 tumor proportion score (TPS) $\geq 1 \%$ : Openlabel, phase 3 KEYNOTE-042 study. J Clin Oncol 2018;36:LBA4.

10. Jotte RM, Cappuzzo F, Vynnychenko I, et al. IMpower131: primary PFS and safety analysis of a randomized phase III study of atezolizumab + carboplatin + paclitaxel or nab-paclitaxel vs carboplatin + nab-paclitaxel as $1 \mathrm{~L}$ therapy in advanced squamous NSCLC. J Clin Oncol 2018;36:LBA9000.

11. Hellmann MD, Ciuleanu TE, Pluzanski A, et al. Nivolumab plus Ipilimumab in Lung Cancer with a High Tumor Mutational Burden. N Engl J Med 2018;378:2093-104.

12. Reck $M$, Schenker $M$, Lee KH, et al. Nivolumab plus ipilimumab versus chemotherapy as first-line treatment in advanced non-small-cell lung cancer with high tumour mutational burden: patient-reported outcomes results from the randomised, open-label, phase III CheckMate 227 trial. Eur J Cancer 2019;116:137-47.

13. Bristol-Myers Squibb Press release 19.10.2018: BristolMyers Squibb Provides Update on the Ongoing Regulatory Review of Opdivo Plus Low-Dose Yervoy in First-Line Lung Cancer Patients with Tumor Mutational Burden $\geq 10 \mathrm{mut} / \mathrm{Mb}$. Available online: https://news.bms. com/press-release/corporatefinancial-news/bristol-myerssquibb-provides-update-ongoing-regulatory-review

14. Hellmann MD, Paz-Ares L, Bernabe Caro R, et al. Nivolumab plus Ipilimumab in Advanced Non-Small-Cell 
Lung Cancer. N Engl J Med 2019;381:2020-31.

15. Paz-Ares L, Ciuleanu TE, Yu X, et al. Nivolumab (NIVO) + platinum-doublet chemotherapy (chemo) vs chemo as first-line (1L) treatment (tx) for advanced non-small cell lung cancer (aNSCLC): CheckMate 227 - part 2 final analysis. Available online: https://www.esmo.org/meetings/ past-meetings/esmo-immuno-oncology-congress-2019/ congress-coverage/news-press-releases/first-linenivolumab-plus-chemotherapy-did-not-meet-theprimary-endpoint-of-overall-survival-in-non-squamousnsclc

Cite this article as: Hockenhull $\mathrm{K}$, Ortega-Franco A, Califano R. Pembrolizumab plus platinum-based chemotherapy for squamous non-small cell lung cancer: the new kid on the block. Transl Lung Cancer Res 2021;10(9):3850-3854. doi: 10.21037/ tlcr-20-715 